\title{
Fast Imaging of Carbon Nanotube Nucleation and Growth Processes using Environmental TEM
}

\author{
Dmitri N. Zakharov ${ }^{1}$, Mostafa Bewedy ${ }^{2}$, Cory Czarnik ${ }^{3}$, A. John Hart ${ }^{2}$, Shigeki Misawa ${ }^{4}$, Eric A. Stach ${ }^{1}$ \\ ${ }^{1}$ Center for Functional Nanomaterials, Brookhaven National Laboratory, Upton, NY 11973, USA \\ ${ }^{2}$ The Laboratory for Manufacturing and Productivity, Massachusetts Institute of Technology, \\ Cambridge, MA 02139 \\ ${ }^{3}$ Gatan, Inc., Pleasanton, CA 94588 \\ ${ }^{4}$ RHIC and ATLAS Computing Facility, Brookhaven National Laboratory, Upton, NY 11973
}

Carbon nanotubes (CNT), an allotrope of carbon, are made out of seamlessly rolled cylindrical sheets of graphene. There are number of possible ways to seamlessly roll a sheet of graphene into a nanotube. Hence, each single walled carbon nanotube (SWCNT) is characterized by a chirality vector. CNTs exhibit varying properties depending on their structure and morphology such as outer and inner diameters, number of walls, length and chirality. As a result it becomes vital to grow tubes with predetermined structure and morphology for any potential application. The typical growth process of CNTs involves iron catalyst particles being deposited over a $\mathrm{SiO}_{2}$ or $\mathrm{Al}_{2} \mathrm{O}_{3}$ substrate, reduced in the presence of hydrogen and exposed to a source of carbon at a temperature. Under hydrocarbon decomposition conditions a CNT nucleates on a catalytic particle and grows via the vapor-liquid-solid (VLS) mechanism. If majority of particles undergo a nucleation process then so called carpet growth is achieved resulting in thick forest of CNTs formed on seeded substrate.

Recent progress in environmental transmission microscopy (ETEM) has allowed us to address several fundamental questions in the field of catalytic carbon nanotube growth. First of all a correlation between particle structure/morphology and SWNT chirality was demonstrated [1] opening, thus, a possibility for chirality control. It was also shown that catalyst particles are always evolving during the CNT growth process [2]. They undergo Ostwald ripening and subsurface diffusion resulting in catalyst mass loss, which turns out to be one of possible ways of CNT termination. Furthermore, it was found that in addition to the role of water as gentle etching agent [3] it responsible for suppression of increasing size of catalyst particles by Ostwald ripening mechanism.

The purpose of this work was to study the collective behavior of nucleated CNTs during the carpet growth. Since a majority of catalytic processes are fast in nature and thus require high speed imaging we have equipped an FEI image corrected Titan 80-300 environmental S/TEM installed at the Center for Functional Nanomaterials at Brookhaven National Laboratory with Gatan K2 in-situ camera. This camera utilize a direct electron detection CMOS chip that has a high resistivity to electron damage and a high sensitivity capable of delivering up to 1600 frames per second.

Samples for electron microscopy were prepared by electron beam assisted deposition of $10 \mathrm{~nm}^{\mathrm{Al}_{2} \mathrm{O}_{3}}$ and then $1 \mathrm{~nm} \mathrm{Fe}$ over $20 \mathrm{~nm} \mathrm{SiO}_{2}$ TEM window. Growth of the initial stage of CNT carpets were conducted inside an ETEM at temperatures of $650^{\circ} \mathrm{C}$ in the presence of acetylene. We have been able to establish the ETEM as a fully functional LP-CVD system for CNT growth and study the early stages of forest nucleation and alignment (Figure 1). We were also studied the role of CNT interactions in the carpet growth termination. 


\section{References:}

[1] A.R. Harutyunyan, G. Chen, T.M. Paronyan, E.M. Pigos, O.A. Kuznetsov, K. Hewaparakrama, S.M.Kim, D. Zakharov, E.A. Stach, G.U. Sumanasekera, "Preferential Growth of Single-Walled Carbon Nanotubes with Metallic Conductivity”, Science 326, no. 5949, pp. 116-120 (2009)

[2] S.M. Kim, C.L. Pint, P.B. Amama, D.N. Zakharov, R.H. Hauge, B. Maruyama, and E.A. Stach, "Evolution in Catalyst Morphology Leads to Carbon Nanotube Growth Termination", J. Phys. Chem.

Lett. 1, pp. 918-922 (2010)

[3] K. Hata, D.N. Futaba, K. Mizuno, T. Namai, M.Yumura, S. Iijima, "Water-Assisted Highly Efficient Synthesis of Impurity-Free Single-Walled Carbon Nanotubes”, Science 306, pp. 1362 (2004)
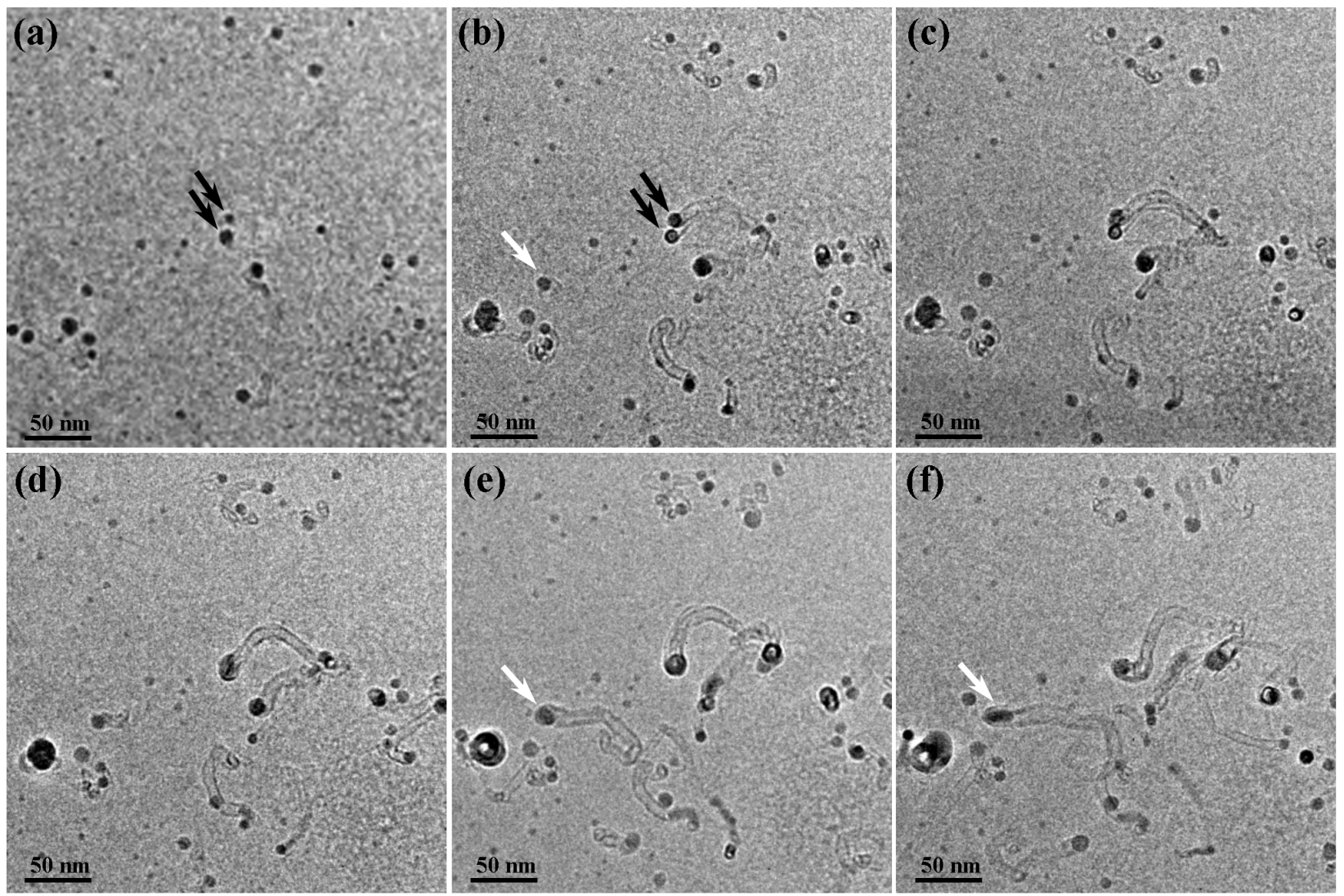

Figure 1. Individual frames (a) - (f) from a movie of initial stages of catalytic carbon nanotube carpet nucleation and growth at $650^{\circ} \mathrm{C}$ inside environmental transmission electron microscope in the presence of acetylene. (a) Two iron particles marked by black arrows. Each is about to nucleate a tube. (b) Same particles as in (a) participate in carbon nanotube growth via VLS mechanism. White arrow points to a catalyst particle that has not nucleate a tube yet. (c) Evolution of two particles marked with black arrows in (b). Particles are closer to each other. (d) Two particles are now coalesced, which results in one tube termination. Other tube continues to grow as seen in (e) and (f). (e) Shows nucleation of a tube on the particle marked by white arrow. (f) Shows change to the particle shape with respect to the (e). 